\title{
Characterization of oscillatory motions in the stable atmosphere of a deep valley
}

\author{
3 Yann Largeron · Chantal Staquet · Charles Chemel
}

revised version, submitted 21 September 2012

\begin{abstract}
In a valley sheltered from strong synoptic effects, the dynamics of the valley atmosphere at night is dominated by katabatic winds. In a stably stratified atmosphere, these winds undergo temporal oscillations, whose frequency is given by $N \sin \alpha$ for an infinitely long slope of constant slope angle $\alpha, N$ being the buoyancy frequency. Such an unsteady flow in a stably stratified atmosphere may also generate internal gravity waves (IGWs). The numerical study by Chemel et al. (Meteorol Atmos Phys 203:187-194, 2009) showed that, in the stable atmosphere of a deep valley, the oscillatory motions associated with the IGWs generated by katabatic winds are distinct from those of the katabatic winds. The IGW frequency was found to be independent of $\alpha$ and about $0.8 \mathrm{~N}$. Their study did not consider the effects of the background stratification and valley geometry on these results. The present work extends this study by investigating those effects for a wide range of stratifications and slope angles, through numerical simulations for a deep valley. The two oscillatory systems are reproduced in the simulations. The frequency of the oscillations of the katabatic winds is found to be equal to $N$ times the sine of the maximum slope angle. Remarkably, the IGW frequency is found to also vary as $C_{w} N$, with $C_{w}$ in the range $0.7-0.95$. These values for $C_{w}$ are similar to those reported for IGWs radiated by any turbulent field with no dominant frequency component. Results suggest that the IGW wavelength is controlled by the valley depth.
\end{abstract}

Keywords Complex terrain - Internal gravity waves - Katabatic winds · Numerical simulations $\cdot$ Stably stratified atmosphere

\section{Introduction} to the heating or cooling of the ground surface. The cooling of the ground surface produces

Y. Largeron · C. Staquet (fi)

LEGI, UJF/CNRS/G-INP, BP 53, 38041 Grenoble cedex 9, France

E-mail: Chantal.Staquet@legi.grenoble-inp.fr

C. Chemel

National Centre for Atmospheric Science (NCAS), Centre for Atmospheric \& Instrumentation Research (CAIR), University of Hertfordshire, College Lane, Hatfield, AL10 9AB, UK 
a shallow layer of cool, stable air above it, inducing downslope flows also referred to as katabatic flows (e.g. Simpson 1994; Poulos and Zhong 2008). As the cold air flows down the slopes, it accumulates over the centre of the valley, thereby leading to a pool or lake of stably stratified air (Mori and Kobayashi 1996; Whiteman et al. 2008).

The characteristics of katabatic winds were first reported from field measurements during summer nights. The winds have a jet-like velocity profile, with maximum of the order of $5 \mathrm{~m} \mathrm{~s}^{-1}$ reached at about $10 \mathrm{~m}$ above the ground, depending upon stratification and surface stress, and a very stable thermal gradient, up to $0.1 \mathrm{~K} \mathrm{~m}^{-1}$ (e.g. Whiteman 2000). Observational studies reported katabatic flows of different nature, from highly turbulent to quasisteady flows, depending on the Richardson number (see for instance Gryning et al. 1985; Helmis and Papadopoulos 1996; Monti et al. 2002; Bastin and Drobinski 2005; Princevac et al. 2008; Viana et al. 2010; Mahrt et al. 2010). A refined classification based on dimensional analysis and momentum balance was proposed by (Mahrt 1982) for stationary flow over a constant slope with simple friction. Whatever their nature, katabatic flows have been shown to undergo temporal oscillations, in both observational and numerical studies (e.g. van Gorsel et al. 2004; Fedorovich and Shapiro 2009). The existence of these oscillations was first accounted for by Fleagle (1950) for an isothermal (and thus stably stratified) atmosphere. As the air flows down the slopes, it undergoes locally a cycle of compressional warming, deceleration (because of the isothermal atmosphere), cooling by the ground surface and further acceleration. McNider (1982) showed, using a simple model coupling the along-slope velocity component and the potential temperature of a fluid particle, that buoyancy effects are responsible for the oscillatory behaviour of the katabatic flows. For an infinitely long slope with a slope angle $\alpha$ (with respect to the horizontal) of constant value and a constant vertical gradient of potential temperature, the model predicts that the frequency of these oscillations is $N \sin \alpha$, where $N$ is the buoyancy (or Brunt-Väisälä) frequency (the square of which is proportional to the vertical gradient of potential temperature).

It is well-known that a body oscillating at frequency $\Omega$ in a stably-stratified fluid with constant $N$ generates internal gravity waves (IGWs) if $\Omega<N$ (e.g. Lighthill 1978). The frequency of these waves (equal to $\Omega$ ) is denoted $\omega_{w}$ hereafter for clarity. These waves have a very peculiar dispersion relation (von Görtler 1943; Mowbray and Rarity 1967): their frequency does not depend on the modulus of the wave vector $\mathbf{k}$ but only on the angle $\phi$ that this wave vector makes with respect to the horizontal. In the absence of rotation, the dispersion relation for IGWs is

$$
\omega_{w}^{2}=N^{2} \cos ^{2} \phi
$$

When IGWs are generated by a turbulent field with no dominant frequency component, observations of the radiated IGW field reveal that the IGWs propagate at a fixed angle with respect to the horizontal, of about $45^{\circ}$. This implies, from the dispersion relation (1), that a very narrow range of frequencies, centred about $0.7 N$, is actually excited (e.g. Wu 1969; Cerasoli 1978; Dohan and Sutherland 2003; Taylor and Sarkar 2007).

Few studies have dealt with the generation of IGWs by katabatic flows (Mori and Kobayashi 1996; Renfrew 2004; Yu and Cai 2006; Princevac et al. 2008; Whiteman et al. 2008; Viana et al. 2010). The numerical study by Chemel et al. (2009) showed that, in the stable atmosphere of an idealized deep valley, the oscillatory motions associated with the IGWs generated by katabatic winds are distinct from those of the katabatic winds. Chemel et al. (2009) also found that the power spectrum of the IGWs is peaked for a ratio $\omega_{w} / N \approx 0.8$, close to that observed in stably stratified turbulence. Only one numerical simulation was considered in this study, and so the generality of the results was not assessed. The aim of the present work is to extend the study of Chemel et al. (2009) by investigating 
the effects of the background stratification of the atmosphere and valley geometry on the characteristics of the IGW field.

For this purpose, we analyse a set of numerical simulations, performed with the Advanced Regional Prediction System (ARPS), for a wide range of stratifications and slope angles. The design of the simulations is described in Sect. 2. The general features of the katabatic and valley winds are briefly reported in Sect. 3 while a detailed analysis of the oscillatory motions is presented in Sect. 4 . The influence of the initial ground surface temperature, background stratification of the atmosphere and valley geometry on the characteristics of the IGW field are discussed in Sect. 5. Conclusions are given in Sect. 6.

\section{Design of the numerical simulations}

\subsection{The numerical model}

The numerical simulations are performed with the ARPS numerical model (Xue et al. 2000). The ARPS model is a non-hydrostatic atmospheric model that is appropriate for scales ranging from a few metres to hundreds of kilometres. The model solves the compressible Navier-Stokes equations, which describe the dynamics of the flow, using a terrain-following coordinate system. It involves surface layer physics and a soil model. In the present study, the air is considered as dry, even though microphysical processes are also included in the ARPS model. Spatial derivatives are discretized with a centered fourth-order finite difference scheme on a staggered grid of Arakawa $\mathrm{C}$ type. Time integration is performed with a centered leapfrog time difference scheme using a mode-splitting time integration technique to deal with the acoustic modes. The turbulent kinetic energy (TKE) 1.5-order closure scheme (Deardorff 1980) is used to model the subgrid scales.

\subsection{The topography of the valley}

The valley is oriented south-north and is connected to a plain to the south so that an alongvalley wind can develop. The analytical expression for the topography of the valley is given by (see for instance Rampanelli et al. 2004)

$$
h(x, y)=H h_{x}(x) h_{y}(y),
$$

where

$$
h_{x}(x)= \begin{cases}1, & |x|>S_{x}+V_{x} \\ \frac{1}{2}-\frac{1}{2} \cos \left(\pi \frac{|x|-V_{x}}{S_{x}}\right), & V_{x} \leq|x| \leq S_{x}+V_{x} \\ 0, & |x|<V_{x}\end{cases}
$$

and

$$
h_{y}(y)=\frac{1}{2}+\frac{1}{2} \tanh \left(\frac{y-y_{0}}{S_{y}}\right)
$$

$H$ is the valley depth, $S_{x}$ and $S_{y}$ are the width of the sloping sidewall along the west-east direction $x$ and south-north direction $y$, respectively, $2 V_{x}$ is the width of the valley floor. Note that $h(x, y)=0$ for $-V_{x}<x<V_{x}$, whatever $y$, implying that the valley floor is flat. The function $h_{y}(y)$, which defines the height of the plateaux along the valley axis, is displayed in Fig. 5. 
We used two sets of values for these parameters, which correspond to the topographies referred to as T1 and T2 hereafter. The topography T1, used in Chemel et al. (2009), is characterized by a valley length of $20 \mathrm{~km}$, with $H=1700 \mathrm{~m}, S_{x}=2640 \mathrm{~m}, S_{y}=5000 \mathrm{~m}$, $V_{x}=620 \mathrm{~m}$ (and $y_{0}=10 \mathrm{~km}$ since $y$ varies between 0 and $20 \mathrm{~km}$ in the present paper) (see Fig. 1a). Defined by this set of parameters, the topography T1 can be considered as an idealized representation of the Chamonix valley, located in the French Alps. It is worth noting that, since the valley depth varies along the valley axis (see Fig. 1a), so does the maximum value of the valley side slope for a given $x$ location. This maximum slope can be calculated from Equations 2 to 4 , yielding $0.5 \pi h_{y}(y) / S_{x}$, corresponding to a slope angle of about $45^{\circ}$ at the valley end. In the following, the words the slope of the topography or, more simply, the slope refers to the slope of the valley side wall.

The topography T2 is used in Sect. 5 to investigate the dependency of the IGW field on the geometry of the valley. The key difference between the topographies T1 and T2 is that the maximum slope angle for the topography T2 is approximately constant, at a value of about $30^{\circ}$, along the valley axis over a distance greater than half of the valley length. The valley length and the parameters $S_{x}$ and $V_{x}$ are the same as for the topography T1 but the sloping sidewall width along $y$ and the valley depth are set to $S_{y}=1200 \mathrm{~m}$ and $H=1000 \mathrm{~m}$, respectively.

\subsection{Model setup}

The model is run for a 3-hour nocturnal situation starting at 2200 UTC (corresponding to time $t=0$ ) in winter at the latitude of the Chamonix valley. No katabatic flow is prescribed at the initial time. For a deep valley under stable conditions, as is the case here, the valley atmosphere is often decoupled from the air above the valley (see for instance Whiteman 2000), and so no synoptic forcing was prescribed as well. The velocity field is thus set to zero in the numerical domain at the initial time.

\subsubsection{The initial stratification}

The initial buoyancy frequency $N$ is set to a constant value, and so the initial vertical gradient of potential temperature $d \theta / d z=\left(\theta_{0} / g\right) N^{2}$ is constant (i.e., the potential temperature increases linearly with height). The value of the reference potential temperature $\theta_{0}$ is set to that of the initial near-surface potential temperature at the valley floor, namely $271 \mathrm{~K}$. (The near-surface temperature is the temperature of the first grid point in the atmosphere above the ground.) Note that, in the study by Chemel et al. (2009), the initial buoyancy frequency profile was derived from measurements in the Riviera valley, located in the Swiss Alps, and varied with height. The constant value of $N$ used in the present work will allow for a sensivity study of the influence of the background stratification upon the IGW dynamics, by varying the initial value of $N$ from $0.91 \times 10^{-2}$ to $2.33 \times 10^{-2} \mathrm{rad} \mathrm{s}^{-1}$, corresponding to an initial stratification $d \theta / d z$ ranging from 2.3 to $15 \mathrm{~K} \mathrm{~km}^{-1}$ (see Table 1). This range of values covers most stable situations encountered in a valley atmosphere.

\subsubsection{The initial ground surface temperature and subsequent evolution}

The temperature of the ground surface $T_{S}$ (namely, the skin-surface temperature) is initialized with an offset from the temperature of the near-surface air $T_{a}$. The offset $T_{s}-T_{a}$ is set to either zero or $-3 \mathrm{~K}$ depending on the simulation (see Table 1). The deep soil temperature 
$T_{2}$ is initialized in a similar way with an offset $T_{2}-T_{a}$ of value either zero or $-5 \mathrm{~K}$ depending on the simulation (see Table 1). Note these conditions are imposed at the initial time only and are therefore not a continuous forcing.

The change of the temperature of the two soil layers with time is governed by a surface energy budget taking into account the radiational cooling of the surface from the emission of longwave radiation. The time evolution of the ground surface temperature $T_{S}$ half way down the slope at $y=15 \mathrm{~km}$ for simulation $\mathrm{S} 1$ is displayed in Fig. 2. $T_{S}$ decreases by a few $\mathrm{K}$ per hour (about $6 \mathrm{~K} \mathrm{~h}^{-1}$ during the first hour of the simulation and $1-2 \mathrm{~K} \mathrm{~h}^{-1}$ afterwards). This rate of cooling is consistent with that derived from in situ measurements at a field site in Vermont, USA, reported by Peck (1996).

\subsubsection{Boundary conditions}

Open boundary conditions are used in the horizontal directions. An impermeability condition is imposed at the ground surface, namely, the velocity component normal to the ground is zero there. A Rayleigh sponge is introduced at the top of the domain in order to absorb upward propagating waves.

The surface roughness length is set to $0.1 \mathrm{~m}$, a value typical of cultivated areas. The Monin-Obukhov surface layer scheme is coupled to the two-layer soil-vegetation model developed by Noilhan and Planton (1989) to provide surface forcing in terms of momentum, heat and moisture fluxes.

\subsection{Numerical parameters}

The domain is discretized using $61 \times 103$ grid points in the horizontal, with a horizontal grid resolution of $200 \mathrm{~m}$. The calculations are made on 140 vertical levels up to $7000 \mathrm{~m}$. The grid mesh is stretched along the vertical to accommodate a high vertical resolution close to the ground surface, of $5 \mathrm{~m}$ below $100 \mathrm{~m}$ and then gradually increasing with height to reach $98 \mathrm{~m}$ at the top of the domain. The time step is $0.25 \mathrm{~s}$.

\section{The katabatic and valley winds for simulation S1}

In this section and the next one, we focus on simulation S1 (see Table 1) for which the initial stratification is in the middle of the range of stratifications considered in our work.

\subsection{The katabatic wind}

\subsubsection{General features of the katabatic wind}

As is customary, we introduce a rotated coordinate system $(s, n)$ where $s$ is the coordinate along the sloping surface, positive down the slope, and $n$ is the coordinate normal to the sloping surface, positive upwards. Note that the grid size along the $n$-axis at a given location along the slope is equal to $d n=\cos \left(\alpha_{l o c}\right) d z$, where $\alpha_{l o c}$ is the angle of the slope at this location. Since $d z=5 \mathrm{~m}$ in the present study, for $\alpha_{l o c}=45^{\circ}$ for instance, $d n \simeq 3.5 \mathrm{~m}$.

The velocity component along the sloping surface, denoted by $u_{s}$, is displayed in Fig. 3 as a function of $n$ for $y=7 \mathrm{~km}$ and $t=74 \mathrm{~min}$, at the location of maximum slope angle, 
equal here to $21^{\circ}$ (i.e., half way down the slope). The katabatic wind is distributed in a layer immediately above the slope, of depth of about $30 \mathrm{~m}$, and reaches a maximum value of $2-3 \mathrm{~m} \mathrm{~s}^{-1}$ at the first grid point above the ground surface (i.e. at $2.5 \mathrm{~m}$ in our calculation). These features are in agreement with in situ measurements of katabatic winds on steep slopes (i.e., with a slope angle larger than $10^{\circ}$ ), either on a single slope (e.g. Helmis and Papadopoulos 1996; Monti et al. 2002) or in a valley (Gryning et al. 1985; van Gorsel et al. 2004). A return flow (of very small amplitude) is created above the downslope flow as a result of mass conservation. Slant observations of such a return flow are available (e.g. Buettner and Thyer 1965) as these require the katabatic wind to flow in a quiet environment and remain quasi-two-dimensional (that is, with no cross-slope wind). Such a return flow has also been observed in numerical simulations of katabatic flow (Skyllingstad 2003; Catalano and Cenedese 2010).

\subsubsection{Temporal oscillations of the katabatic wind}

At a given location along the slope, the along-slope component of the wind $u_{s}$ varies with time. This is attested in Fig. 4a where $u_{s}$ is plotted near the bottom of the slope: $u_{s}$ undergoes oscillations about a positive value of approximately $0.5 \mathrm{~m} \mathrm{~s}^{-1}$, with an apparently well-defined period. These oscillations are present all along the slope. For an infinitely long slope of constant angle $\alpha$ and for a constant buoyancy frequency $N$, the frequency of these oscillations is given by (McNider 1982)

$$
\omega_{k}=N \sin \alpha
$$

For a given $y$ location along the valley axis, $\alpha$ varies along the slope, and so it is not obvious which value should be used for $\alpha$ in Equation 5. The frequency spectrum associated with the time series of $u_{s}$ displayed in Fig. 4a shows a dominant peak with a period of $10 \mathrm{~min}$ (see Fig. 4b). Using the value of $N$ for simulation S1, Equation 5 yields a value for $\alpha$ of about $45^{\circ}$, which corresponds to the maximum angle of the slope at this $y$ location. Hence, the period of the oscillations would be set by the background stratification and maximum angle of the slope. We show below that this result also holds for a more gentle slope.

In parts of the valley where the slope angle is smaller, the period of these oscillations becomes longer, in agreement with Equation 5. The frequency spectrum of $u_{s}$ at the same $x$ location as Fig. 4a but for $y=7 \mathrm{~km}$ (i.e., at a location closer to the valley mouth), shows a dominant peak for a period of $20 \mathrm{~min}$ (see Fig. 4c). For this period, Equation 5 leads to $\alpha=21^{\circ}$, which is the maximum slope angle at that $y$ location.

Note that the frequency spectra displayed in Fig. 4b and Fig. 4c show several peaks, the magnitudes of which vary with distance down the slope. It is still remarkable that the fluid particle model developed by McNider (1982) for an infinitely long slope with constant slope angle and a constant buoyancy frequency, predicts the dominant peak of these frequency spectra.

The oscillations of the katabatic wind have been mainly detected in a shallow layer immediately above the slopes, of depth 20 to $30 \mathrm{~m}$. Having said that, oscillations have also been detected in the return flow and have a frequency close to that of the downslope flow (not shown). This indicates that the return flow is tightly coupled to the downslope flow. 
3.2 The valley wind

As a result of downslope flows filling the valley with cold air, the valley atmosphere cools faster than the plain atmosphere at the same altitude, producing higher pressure in the valley compared to the plain. This pressure gradient drives a down-valley flow from the valley to the plain (see Fig. 5). As the cold air flowing down the slopes accumulates over the centre of the valley, the valley temperature inversion grows deeper and deeper. As the inversion layer deepens, the layer of down-valley wind deepens. As can be seen in Fig. 5, the depth of the inversion layer reaches that of the valley $45 \mathrm{~min}$ into the simulation. After the period of rapid growth of the valley inversion, the down-valley wind prevails through the depth of the fully developed inversion. It displays a minimum value of $-0.5 \mathrm{~m} \mathrm{~s}^{-1}$ close to the ground, in a $40 \mathrm{~m}$ deep layer occuring close to the mouth of the valley.

The above description of the wind and temperature structure evolution in the valley agrees well with observations and can be found in textbooks (e.g. Whiteman 2000). However, in our work, the establishment of the down-valley wind is a bit more subtle. Because the height of the plateaus and slope angle of the valley sidewalls increase with distance from the plain, the volume of air pouring down the slopes is larger as one moves toward the valley end. This results in an along-valley pressure gradient, which accelerates the flow.

\section{The internal gravity wave field for simulation S1}

\subsection{Emission of the internal gravity wave field}

Since the atmosphere is stably stratified, any non-horizontal perturbation varying in time with a frequency component smaller than $N$, generates IGWs. As shown in Sect. 3, katabatic winds are unsteady, with a frequency spectrum containing frequency components smaller than $N$ (e.g. $\omega_{k}$ ), and so should emit an IGW field propagating away from the slopes.

Let us show that Coriolis effects do not affect the wave dynamics. Once emitted, the wave dynamics can be assumed to be linear and, therefore, satisfy the dispersion relation. Accounting for Coriolis effects, relation (1) becomes $\omega_{w}^{2}=N^{2} \cos ^{2} \phi+f^{2} \sin ^{2} \phi$, which can also be written as $\left(\omega_{w} / N\right)^{2}=\cos ^{2} \phi+(f / N)^{2} \sin ^{2} \phi$. For $\phi$ smaller than $\pi / 2$, the second term of this dispersion relation can be neglected if $f / N \ll 1$. In the present case $f \simeq 10^{-4}$ $\operatorname{rad~s}^{-1}$ and $N=1.47 \times 10^{-2} \mathrm{rad} \mathrm{s}^{-1}$ (so that $f / N \simeq 0.007$ ) implying that rotation effects can be ignored. As discussed in classical textbooks (e.g Lighthill 1978), in the absence of rotation, the flow induced by plane IGWs is a parallel shear flow, where the velocity is normal to the wave vector and lies in the same vertical plane. Hence the angle of the velocity vector with respect to the vertical is the angle $\phi$ in the dispersion relation (1).

The emission of IGWs by the unsteady katabatic winds is illustrated in Fig. 6b, in which the vertical velocity component $w$ is displayed in a vertical cross section for $y=15 \mathrm{~km}$ and $t=45 \mathrm{~min}$. Since the generation of IGWs has just started (i.e., the wave-induced velocity is zero away from the slopes), the signature of the IGW field appears as upward and downward motions and resembles closed cells. The same feature was found in the numerical study by Renfrew (2004) for the IGW field generated by an unsteady (decelerating) katabatic flow on a slope-varying ice shelf (see Fig. 14 of this paper), and by Catalano and Cenedese (2010) when analysing nocturnal conditions in a valley of constant slope. A remarkable feature of this cell pattern is that the angle that the cell axis makes with the vertical is nearly constant along the valley sidewalls, despite the varying slope angle. The cell axis angle is $\phi$ which implies that the IGW frequency is constant for $N$ constant (see the dispersion relation (1)) 
and independent of the slope angle. This important finding is further discussed in the next sections. The maximum value of the wave-induced vertical velocity is about $0.2 \mathrm{~m} \mathrm{~s}^{-1}$, that is, one order of magnitude smaller than the vertical velocity of the katabatic flows, which emits the waves. (Indeed, using the relation $w=(\sin \alpha) u_{s}$ between the vertical and alongslope components of the velocity, one finds, for $\alpha=45^{\circ}$ and from Fig. 3, that the maximum amplitude of $w$ is about $1.5 \mathrm{~m} \mathrm{~s}^{-1}$.)

Since the IGWs are generated by the katabatic flow, this flow needs to become established before the IGWs become apparent. For the conditions of simulation S1, about $20 \mathrm{~min}$ are required before IGWs can be observed. The wave field first appears at the bottom of the slopes (see Fig. 6a), as also found by Renfrew (2004) and Yu and Cai (2006). The latter authors, who conducted a numerical study similar to that of Renfrew (2004), focused on the most likely origin of the IGW field, namely the vertical velocity perturbation induced by a hydraulic jump in the katabatic flow. This hydraulic jump can result from the katabatic flow encountering a cold pool at the bottom of the valley or, simply, strongly decelerating as it reaches the foot of the slope. The possible occurence of a hydraulic jump is usually estimated by computing a Froude number associated with the katabatic flow. Using the classical definition (e.g. Ball 1956) $F r=U /\left[\left(g \Delta \theta / \theta_{0}\right) H\right]$, where $U$ is a typical velocity of the katabatic flow and $\Delta \theta$ the potential temperature deficit across the katabatic wind layer of height $H$, one finds $F r \simeq 2-3$ for simulation S1. Therefore $F r>1$ for the katabatic flow (and $F r<1$ downstream of that flow) so that a hydraulic jump is likely to occur. In the present case, this hydraulic jump would be created by the katabatic flow encountering the flat valley floor.

Fig. 6 shows that a standing IGW pattern is created at the bottom of the valley because of the convergence of the katabatic flows originating from the slopes of each valley sidewall. Finally, we note that the IGWs first form along the longest and steepest slopes (i.e, as one moves toward the valley end) and, later in time, along shorter and shallower slopes as well (see also Section 4.3 and Fig. 8a).

\subsection{Frequency analysis of the internal gravity wave field}

The purpose of this section is to show that a quasi-monochromatic IGW field develops, the frequency of which $\omega_{w}$ is independent of the frequency $\omega_{k}$ of the oscillations of the katabatic flows. In the following, we therefore analyse how $\omega_{w}$ varies with $y$ (i.e., along the valley axis). The IGW frequency $\omega_{w}$ is determined by the dominant peak of the vertical velocity frequency spectrum computed at a location well above the katabatic flows. Note that, for an altitude lower than $500 \mathrm{~m}$ above the ground surface, because of the standing IGW system that develops and of possible wave-wave interactions, no clear frequency can be identified in the spectrum. Above $500 \mathrm{~m}$ by contrast, any altitude may be chosen to compute this spectrum; indeed, since the wave field propagates in a homogeneous medium, the wave frequency does not vary with $z$ (as we checked it).

The frequency spectrum of the vertical velocity $w$ was computed for $x=-0.6 \mathrm{~km}$ at an altitude of $z=2200 \mathrm{~m}$ (i.e., above the height of the plateaus), for different values of $y$ along the valley axis. We recall that the maximum slope angle of the topography $\alpha_{\max }$ in a given vertical $(x, z)$ plane varies with $y$, and so does the frequency $\omega_{k}=N \sin \alpha_{\max }$ of the oscillations of the katabatic winds. The ratios $\omega_{w} / N$ and $\omega_{k} / N$ computed at this $(x, z)$ location are plotted versus $y$ in Fig 7a. The ratio $\omega_{w} / N$ appears to be independent of $y$ and therefore of the slope angle. Hence, the IGW frequency does not seem to be imposed by the frequency $\omega_{k}$ of the oscillations of the katabatic winds. More precisely, $\omega_{w}$ varies as $C_{w} N$, 
with $C_{w}$ in the range $0.7-0.95$. A representative value of $C_{w}$ may be taken as its $y$-average value, equal to 0.82 .

Note also that, for steeper slopes, the values of $\omega_{w}$ are closer to that of $\omega_{k}$ because $\omega_{k}$ increases with $\alpha_{\max }$ so that the absence of relation between $\omega_{w}$ and $\omega_{k}$ is more difficult to assess there.

\subsection{Wavelength of the internal gravity wave field}

One striking feature of the dispersion relation (1) is that only a time scale comes into play, namely $1 / N$, but no length scale. Nevertheless, IGWs usually develop with a well-defined wavelength, which is imposed by an external length scale. This length scale may be fixed by the geometry of the forcing, as for lee waves, or from dimensions of the reservoir which contains the IGWs, as for seiches in lakes. In this section, we compute the wavelengths of the IGW field in all three directions and attempt to determine the external length scale which sets them.

The wavelength can be determined by plotting contours of the vertical velocity field (or potential temperature field) in a time-space diagram (also referred to as a Hovmöller diagram). For a monochromatic wave field, a $\left(t, x_{i}\right)$ diagram provides the phase speed $c_{i}$ in direction $x_{i}$, given by the slope of the contours, as well as the wave period $T_{w}$ and the wavelength $\lambda_{i}$ in that direction. We recall that $c_{i}=\lambda_{i} / T_{w}$, with $T_{w}=2 \pi / \omega_{w}$.

\section{Wavelength along the valley axis}

Contours of the vertical velocity field $w$ in a $(t, y)$ diagram are plotted in Fig. 8a for $x=$ $-0.6 \mathrm{~km}$ at a height of $800 \mathrm{~m}$ above the ground surface for the first $80 \mathrm{~min}$ of simulation. The IGWs reach this height at about $40 \mathrm{~min}$ into the simulation. The slope of the contours, equal to the phase speed $c_{y}$, is infinite for $y$ larger than about $7 \mathrm{~km}$ (i.e., within the valley). Since the IGW period $T_{w}$ is finite, this implies that the wavelength along the $y$ direction is infinite for $y$ larger than $7 \mathrm{~km}$. Therefore, the IGW field may be assumed to be twodimensional (i.e., the IGWs propagate in the $(x, z)$ plane) beyond this distance.

Fig. 8a also clearly shows that IGWs are first emitted toward the valley end, along the longest and steepest slopes (for $y$ larger than about $15 \mathrm{~km}$ ), the wave emission progressively extending to the shallower slopes with time.

\section{Wavelength along the vertical}

Contours of the vertical velocity field $w$ in a $(t, z)$ diagram are plotted in Fig. $8 \mathrm{~b}$ for $x=-0.6 \mathrm{~km}$ and $y=15 \mathrm{~km}$ for the first $80 \mathrm{~min}$ of simulation. As above, phase lines are clearly visible, with a well defined slope $c_{z}$ in the upper part of the phase lines. The value of this slope is equal to $-2.5 \mathrm{~m} \mathrm{~s}^{-1}$. The IGW period $T_{w}$ is given by the distance along the horizontal axis between two maxima of vertical velocity; one finds $T_{w} \approx 10 \mathrm{~min}$, consistent with the value of $\omega_{w} \simeq 0.8 N$ with $N=0.0147 \mathrm{rad} \mathrm{s}^{-1}$. With $\lambda_{z}=c_{z} T_{w}$, one gets $\lambda_{z} \approx 1300 \mathrm{~m}$. Similar phase speed and period are found for any $y>7 \mathrm{~km}$. Since the maximum height of the surrounding plateaus along the valley axis $H$ is equal to $1700 \mathrm{~m}$, one may conclude that $\lambda_{z}$ is set by the valley depth. 
Wavelength along the cross-valley direction

The wavelength along the cross-valley direction $\lambda_{x}$ is simply inferred from $\tan \phi=\lambda_{x} / \lambda_{z}$. The relation $\omega_{w} \simeq 0.8 N$ yields $\phi \simeq 37^{\circ}$ (since $\left.\cos \phi=0.8\right)$ so that $\lambda_{x} \approx 0.75 \lambda_{z} \approx 1000$ $\mathrm{m}$. We note that $\lambda_{x}$ is close to the width of the valley floor $2 V_{x}=1240 \mathrm{~m}$ for the topography T1. As an alternate route to find the external length scale which controls the wavelength, one may conclude that $\lambda_{x}$ is fixed by the width of the valley floor; $\lambda_{z}$ would then result from the dispersion relation (1). However, Chemel et al. (2009) found that $\lambda_{x}$ remains unchanged when the width of the valley floor is doubled, the height of the surrounding plateaus being unchanged. Hence, the results suggest that $\lambda_{z}$ is set by the valley depth, $\lambda_{z}$ imposing $\lambda_{x}$ through the dispersion relation (1) with $\omega_{w} \simeq 0.8 \mathrm{~N}$.

\section{Sensitivity experiments}

In the previous section, we found that the IGW frequency $\omega_{w}$ is equal to $C_{w} N$, with $C_{w}$ in the range $0.7-0.95$, and is independent of the frequency $\omega_{k}$ of the oscillations of the katabatic winds. The purpose of this section is to investigate the effects of the background stratification of the atmosphere and valley geometry on the characteristics of the IGW field. We first show that the simulations used for the sensitivity experiments can be run more efficiently by slightly changing the initial condition in the soil layers.

\subsection{Influence of the initial ground surface temperature}

In simulation $\mathrm{S} 1$, the initial temperature of the ground surface $T_{S}$ was the same as those of the near-surface air $T_{a}$ and deep soil $T_{2}$ (see Table 1). Our hypothesis is that, if $T_{2}$ is lower than $T_{a}$ at the initial time, the katabatic flows should be established faster, and so the IGWs should develop more rapidly. In order to test this hypothesis, we initialized $T_{S}$ and $T_{2}$ in simulation $\mathrm{S} 1$ such as $T_{s}-T_{a}=-3 \mathrm{~K}$ and $T_{2}-T_{a}=-5 \mathrm{~K}$ (see simulation S5 in Table 1). By doing so, the ground surface temperature cools faster, and so does the near-surface air (not shown). As a result, the response of the atmosphere to the surface cooling is more rapid in simulation S5 than in simulation S1 but is qualitatively the same. More precisely, Fig. 9 shows that results of simulation S1 corresponds to those of simulation S5 but delayed by 20 min. To save computing time, all the simulations used for the sensitivity experiments were performed with the same initialization of the ground surface and deep soil temperatures as simulation S5 (see Table 1).

\subsection{Influence of the background stratification}

In order to investigate the effects of the background stratification of the atmosphere, we performed 8 simulations (simulations $S 2$ to $S$ 9, see Table 1) with different values of the buoyancy frequency, ranging from $0.91 \times 10^{-2}$ to $2.33 \times 10^{-2} \mathrm{rad} \mathrm{s}^{-1}$, corresponding to an initial stratification $d \theta / d z$ ranging from 2.3 to $15 \mathrm{~K} \mathrm{~km}^{-1}$.

In agreement with the results of Sect. 4.2, the IGW frequency $\omega_{w}$ is found to be independent on $y$ and therefore on the slope angle for every simulation. We shall therefore assume that the IGW frequency is nearly constant along $y$ and compute its $y$-averaged value, $\left\langle\omega_{w}\right\rangle$, for each value of $N$, at a given $(x, z)$ location (as before, for $x=-0.6 \mathrm{~km}$ and $z=2200 \mathrm{~m}$ ). 
The ratio $\left\langle\omega_{w}\right\rangle / N$ is plotted in Fig. 10 for each value of $N$, in the simulations S2 to S9. Consistent with Sect. 4.2, $\left\langle\omega_{w}\right\rangle$ varies as $C_{w} N$, with $C_{w}$ in the range $0.7-0.95$. The figure also shows that $C_{w}$ is approximately constant and about 0.8 for weak stratification $(d \theta / d z \leq 6$ $\mathrm{K} \mathrm{km}^{-1}$ ) and slightly decreases with $N$ when the stratification becomes stronger.

\subsection{Influence of the topography}

Coincidentally, for the topography $\mathrm{T} 1$ used in simulations $\mathrm{S} 1$ to $\mathrm{S} 9$, the maximum slope angle $\alpha_{\max }$ is about $45^{\circ}$ at the valley end, and so $\sin \alpha_{\max } \approx 0.7$, which is close to the ratio $\omega_{w} / N \approx 0.8$ found hitherto for the IGW field. In order to remove any doubt concerning the non dependence of $\omega_{w} / N$ on $\alpha_{\max }$ (and thus on $\omega_{k}$ ), a simulation was performed with the topography T2, for which the maximum slope angle is approximately constant, at a value of about $30^{\circ}$ (see Sect. 2.2).

The characteristics of the IGW field are in line with the results of Sect. 4. The frequency spectrum associated with the time series of $u_{s}$ at $x=-1.2 \mathrm{~km}$ and $y=15 \mathrm{~km}$ at $12.5 \mathrm{~m}$ above the ground surface (see Fig. 11a) shows that the katabatic winds undergo temporal oscillations at a frequency $\omega_{k}=6.4 \times 10^{-3} \mathrm{rad} \mathrm{s}^{-1}$, giving $\omega_{k} / N \approx 0.5\left(\right.$ namely $\left.\sin 30^{\circ}\right)$. The frequency spectrum associated with the time series of $w$ at $x=-1.2 \mathrm{~km}$ and $y=$ $15 \mathrm{~km}$ at $4000 \mathrm{~m}$ above the ground surface (see Fig. 11b) exhibits a peak at frequency $\omega_{w}=11.8 \times 10^{-3} \mathrm{rad} \mathrm{s}^{-1}$, giving $\omega_{w} / N \approx 0.8$. Thus, we can conclude with no doubt that the frequency $\omega_{w}$ of the IGWs is distinct from that of the oscillations of the katabatic winds $\omega_{k}$ and that the ratio $\omega_{w} / N$ is independent of the valley geometry.

\section{Conclusions}

The purpose of this work was to extend the study by Chemel et al. (2009) by investigating the effects of the background stratification and valley geometry on the characteristics of the IGW field generated by katabatic winds in a deep valley. For this purpose, a set of numerical simulations, for a wide range of stratifications and slope angles, were run for 3 hours during a winter night at mid-latitude and analysed.

The present study confirms that two oscillatory systems, spatially decoupled, coexist, consisting of (i) along-slope temporal oscillations of the katabatic winds and (ii) oscillations associated with the IGW field emitted by the katabatic winds, which propagates away from the slopes. The frequency of the oscillations of the katabatic winds $\omega_{k}$ is found to be equal to $N$ times the sine of the maximum slope angle. The IGW frequency $\omega_{w}$ is found to be independent of $\alpha$ and about $0.8 \mathrm{~N}$.

The novelty here is to analyse the generation of the IGW field and the variations of the IGW frequency and wavelength in space, and as a function of the stratification and valley geometry.

The IGWs first form at the bottom of the slopes, as a result of a hydraulic jump in the katabatic flow, and are then emitted all along the slope. The IGWs are generated along the longest and steepest slopes (i.e, as one moves toward the valley end) and, later in time, along shorter and shallower slopes as well. The IGW field propagates in a plane perpendicular to the valley axis and is therefore two-dimensional. Whatever the location in the valley atmosphere (away from the katabatic flow), its frequency $\omega_{w}$ varies as $C_{w} N$, with $C_{w}$ in the range $0.7-0.95$. The simulations used for the sensitivity experiments with different values of background stratification indicated that the ratio $\omega_{w} / N$ is constant and about 0.8 for weak 
stratification $\left(d \theta / d z \leq 6 \mathrm{~K} \mathrm{~km}^{-1}\right)$ and slightly decreases with $N$ when the stratification becomes stronger. The present analysis also showed that the ratio $\omega_{w} / N$ is independent of the valley geometry and that the IGW wavelength is controlled by the valley depth.

The fact that the ratio $\omega_{w} / N$ varies in a narrow range of values around 0.8 may be explained by the theoretical work of Voisin et al. (2011) (see also Voisin (2007)). This work shows that the power of the IGWs radiated by an oscillating sphere or cylinder displays a maximum value for an oscillating frequency close to $0.8 \mathrm{~N}$ (the radiated power is the wave energy averaged over the period of the oscillating source, divided by the period). When an unsteady flow, such as a katabatic flow, is considered instead of an oscillating sphere, one may argue that this flow possesses a large range of frequencies, among them those with a frequency close to $0.8 \mathrm{~N}$ are the most powerful at emitting IGWs, and therefore dominate the IGW signal. Laboratory experiments of localized turbulence in a stably stratified fluid are consistent with this result, reporting that the IGWs propagate at a fixed angle with respect to the horizontal, of about $45^{\circ}$ (e.g. Wu 1969; Cerasoli 1978; Dohan and Sutherland 2003; Taylor and Sarkar 2007).

Acknowledgements The PhD grant of YL was funded by the French Région Rhône-Alpes as part of the 'Cluster Environnement'. YL and CS also acknowledge financial support from the French 'Institut national des sciences de l'Univers' (INSU) as part of the LEFE/IDAO program. Time-consuming computations were performed thanks to the French IDRIS national supercomputing facilities.

\section{References}

Ball FK (1956) The theory of strong katabatic winds. Austr J Phys 9:373-386

Bastin S, Drobinski P (2005) Temperature and wind velocity oscillations along a gentle slope during seabreeze events. Boundary-Layer Meteorol 114:573-594

Buettner KJK, Thyer N (1965) Valley winds in the mount rainier area. Arch Meteor Geophys Bioklimatol 14:125-147

Catalano F, Cenedese A (2010) High-resolution numerical modeling of thermally driven slope winds in a valley with strong capping. J Appl Meteorol Climatol 49:1859-1880

Cerasoli CP (1978) Experiments on buoyant-parcel motion and the generation of internal gravity waves. J Fluid Mech 86:247-271

Chemel C, Staquet C, Largeron Y (2009) Generation of internal gravity waves by a katabatic wind in an idealized alpine valley. Meteorol Atmos Phys 103:187-194

Deardorff JW (1980) Stratocumulus-capped mixed layers derived from a three-dimensional model. Boundary-Layer Meteorol 18:495-527

Dohan K, Sutherland BR (2003) Internal waves generated from a turbulent mixed region. Phys Fluids 15:488498

Fedorovich E, Shapiro A (2009) Structure of numerically simulated katabatic and anabatic flows along steep slopes. Acta Geophys 57:981-1010

Fleagle RG (1950) A theory of air drainage. J Meteor 7:227-232

van Gorsel E, Vogt R, Christen A, Rotach M (2004) Low frequency temperature and velocity oscillations in katabatic winds. In: Proc. of the 27th International Conference on Alpine Meteorology, 19-23 May 2003, Brig, Switzerland

von Görtler H (1943) über eine schwingungserscheinung in flüssigkeiten mit stabiler dichteschichtung. $\mathrm{Z}$ angew Math Mech 23:65-71

Gryning SE, Mahrt L, Larsen S (1985) Oscillating nocturnal slope flow in a coastal valley. Tellus 37A:196203

Helmis CG, Papadopoulos KH (1996) Some aspects of the variation with time of katabatic flow over simple slope. Q J R Meteorol Soc 122:595-610

Lighthill JM (1978) Waves in Fluids. Cambridge University Press, Cambridge, UK, 504 pp.

Mahrt L (1982) Momentum balance of gravity flows. J Atmos Sci 39:2701-2711

Mahrt L, Richardson S, Seaman N, Stauffer D (2010) Non-stationary drainage flows and motions in the cold pool. Tellus A 62:698-705 
McNider RT (1982) A note on velocity fluctuations in drainage flows. J Atmos Sci 39:1658-1660

Monti P, Fernando HJS, Princevac M, Chan WC, Kowalewski TA, Pardyjak ER (2002) Observations of flow and turbulence in the nocturnal boundary layer over a slope. J Atmos Sci 59:2513-2534

Mori M, Kobayashi T (1996) Dynamic interaction between observed nocturnal drainage winds and a cold air lake. J Meteorol Soc Jpn 74:247-258

Mowbray DE, Rarity DSH (1967) The internal wave pattern produced by a sphere moving vertically in a density stratified liquid. J Fluid Mech 30:489-495

Noilhan J, Planton S (1989) A simple parametrization of land surface processes for meteorological models. Mon Weather Rev 117:536-549

Peck L (1996) Temporal and spatial fluctuations in ground cover surface temperature at a Northern New England site. Atmos Res 41:131-160

Poulos G, Zhong S (2008) An observational history of small-scale katabatic winds in mid-latitudes. Geography Compass 2:10.1111/j.1749-8198.2008.00,166.x

Princevac M, Hunt J, Fernando HJS (2008) Slopes in wide valleys: hydraulic theory and observations. J Atmos Sci 65:627-643

Rampanelli G, Zardi D, Rotunno R (2004) Mechanism of up-valley winds. J Atmos Sci 61:3097-3111

Renfrew IA (2004) The dynamics of idealized katabatic flow over a moderate slope and ice shelf. Q J R Meteorol Soc 130:1023-1045

Simpson JE (1994) Sea Breeze and Local Winds. Cambridge University Press, Cambridge, UK, 234 pp.

Skyllingstad ED (2003) Large-eddy simulation of katabatic flows. Boundary-Layer Meteorol 106:217-243

Taylor JR, Sarkar S (2007) Internal gravity waves generated by a turbulent bottom ekman layer. J Fluid Mech 590:331-354

Viana S, Terradellas E, Yagüe C (2010) Analysis of gravity waves generated at the top of a drainage flow. J Atmos Sci 67:3949-3966

Voisin B (2007) Added mass effects on internal wave generation. In: Proc. of the Fifth International Symposium on Environmental Hydraulics, 4-7 Dec. 2007, Tempe, AZ, USA

Voisin B, Ermanyuk VE, Flor JB (2011) Internal wave generation by oscillation of a sphere, with application sto internal tides. J Fluid Mech 666:308-357

Whiteman CD (2000) Mountain Meteorology: Fundamentals and Applications. Oxford University Press, New York, NY, USA, $355 \mathrm{pp}$.

Whiteman CD, Muschinski A, Zhong S, Fritts D, Hocj SW, Hahnenbreg M, Yao W, Hohreiter V, Behn M, Cheon Y, Clements CB, Horst TW, Brown WOJ, Oncley SP (2008) METCRAX 2006: Meteorological Experiments in Arizona's Meteor Crater. B Am Meteorol Soc 89:1665-1680

Wu J (1969) Mixed region collapse with internal wave generation in a density-stratified medium. J Fluid Mech 35:531-544

Xue M, Droegemeier KK, Wong V (2000) The Advanced Regional Prediction System (ARPS) - a multi-scale non hydrostatic atmospheric simulation and prediction model. Part I: Model dynamics and verification. Meteorol Atmos Phys 75:161-193

Yu Y, Cai XM (2006) Structure and dynamics of katabatic flow jumps: idealised simulations. BoundaryLayer Meteorol 118:527-555 


\section{List of Figures}

1 Topography of the valleys (a) T1 and (b) T2 (see Section 2.2 for details). Note that the valley floor is flat . . . . . . . . . . . . . . .

2 Time series of the ground surface temperature $T_{S}$ half way down the slope at $y=15 \mathrm{~km}$ for simulation $\mathrm{S} 1 \ldots \ldots \ldots \ldots$

3 Along-slope component $u_{s}$ of the katabatic wind versus distance along the vector normal to the sloping surface, for $y=7 \mathrm{~km}$ and $t=74 \mathrm{~min}$, at the $x$ location of maximum slope angle (i.e., half way down the slope), for simulation $\mathrm{S} 1 \ldots \ldots \ldots \ldots \ldots$

4 For simulation S1: (a) Time series of the along-slope component of the wind $u_{s}$ at $x=-1.2 \mathrm{~km}$ and $y=15 \mathrm{~km}$ at $12.5 \mathrm{~m}$ above the ground surface; (b) Frequency velocity spectrum $S\left[u_{S}\right]$ of $u_{s}$ at the same location; (c) Frequency velocity spectrum $S\left[u_{s}\right]$ of $u_{s}$ at $x=-1.2 \mathrm{~km}$ and $y=7 \mathrm{~km}$ at $12.5 \mathrm{~m}$ above the ground surface. The blue dashed line in plots (b) and (c) indicates the pe$\operatorname{riod} 2 \pi / \omega_{k}$ of the oscillations of the katabatic wind calculated using Equation 5 (see text for details) . . . . . . . . . . . . . . . . .

5 For simulation $\mathrm{S} 1$ : colour-filled contours of the along-valley component $v$ of the wind, in $\mathrm{m} \mathrm{s}^{-1}$, in a $(y, z)$ vertical cross section for $x=0 \mathrm{~km}$ and $t=45 \mathrm{~min}$. The black solid line indicates the height of the plateaus along the valley axis . . . . . . . . . . . . . . . .

6 For simulation S1: colour-filled contours of the vertical velocity component $w$, in $\mathrm{m} \mathrm{s}^{-1}$, in a vertical cross section for $y=15 \mathrm{~km}$ at (a) $t=25 \mathrm{~min}$ and (b) $t=45 \min \ldots \ldots \ldots \ldots \ldots \ldots$

7 For simulation S1: ratio of the IGW frequency $\omega_{w}$ to the buoyancy frequency $N(*)$, and of the frequency of the oscillations of the katabatic wind $\omega_{k}$ to $N$ (blue dashed line) versus $y$ (i.e., along the valley axis) for $x=-0.6 \mathrm{~km}$ at an altitude of $2200 \mathrm{~m} \ldots \ldots \ldots \ldots \ldots$

8 For simulation S1: colour-filled contours of the vertical velocity component $w$, in $\mathrm{m} \mathrm{s}^{-1}$, (a) in a $(t, y)$ diagram for $x=-0.6 \mathrm{~km}$ at a height of $800 \mathrm{~m}$ above the valley floor and (b) in a $(t, z)$ diagram for $x=-0.6 \mathrm{~km}$ and $y=$

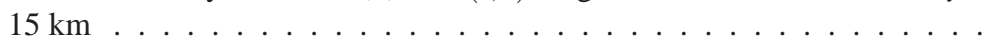

9 Colour-filled contours of the vertical velocity component $w$, in $\mathrm{m} \mathrm{s}^{-1}$, in a $(t, z)$ diagram for $x=-0.6 \mathrm{~km}$ and $y=15 \mathrm{~km}$ (a) for simulation S1 and (b) for simulation $\mathrm{S} 5 \ldots \ldots \ldots \ldots \ldots$

10 Ratio of the mean value of the IGW frequency $\omega_{w}$ over $y$ (i.e., along the valley axis), denoted by $\left\langle\omega_{w}\right\rangle$, and the buoyancy frequency $N(*)$, for $x=$ $-0.6 \mathrm{~km}$ at an altitude of $2200 \mathrm{~m}$, for each value of $N$ in the simulations S2

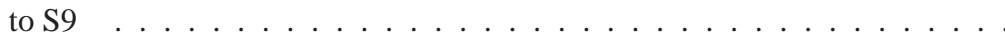

11 For simulation S10: (a) Frequency velocity spectrum $S\left[u_{S}\right]$ of the alongslope component of the wind $u_{S}$ at $x=-1.2 \mathrm{~km}$ and $y=15 \mathrm{~km}$ at $12.5 \mathrm{~m}$ above the ground surface; (b) Frequency velocity spectrum $S[w]$ of the vertical component of the wind $w$ at $x=-1.2 \mathrm{~km}$ and $y=15 \mathrm{~km}$ at $4000 \mathrm{~m}$ above the ground surface. The blue dashed line in plot (a) indicates the frequency of the oscillations of the katabatic winds $\omega_{k}$ calculated using Equation 5. The red dashed line in plot $(\mathbf{b})$ indicates the IGW frequency $\omega_{w} \approx 0.8 \mathrm{~N}$. . 


\section{Tables}

Table 1 Description of the simulations. $T_{s}$ and $T_{a}$ are the temperature of the ground surface and the nearsurface air, respectively, and $T_{2}$ is the deep soil temperature; $N$ is the buoyancy frequency. All values are initial values.

\begin{tabular}{lccccc}
\hline Simulation & $T_{s}-T_{a}(\mathrm{~K})$ & $T_{2}-T_{a}(\mathrm{~K})$ & $d \theta / d z\left(\mathrm{~K} \mathrm{~km}^{-1}\right)$ & $N\left(\mathrm{rad} \mathrm{s}^{-1}\right)$ & Topography \\
\hline S1 & 0 & 0 & 6.0 & $1.47 \times 10^{-2}$ & $\mathrm{~T} 1$ \\
S2 & -3 & -5 & 2.3 & $0.91 \times 10^{-2}$ & $\mathrm{~T} 1$ \\
S3 & -3 & -5 & 3.4 & $1.11 \times 10^{-2}$ & $\mathrm{~T} 1$ \\
S4 & -3 & -5 & 4.7 & $1.30 \times 10^{-2}$ & $\mathrm{~T} 1$ \\
S5 & -3 & -5 & 6.0 & $1.47 \times 10^{-2}$ & $\mathrm{~T} 1$ \\
S6 & -3 & -5 & -5.0 & $1.70 \times 10^{-2}$ & $\mathrm{~T} 1$ \\
S7 & -3 & -5 & 10.0 & $1.90 \times 10^{-2}$ & $\mathrm{~T} 1$ \\
S8 & -3 & -5 & 15.3 & $2.11 \times 10^{-2}$ & $\mathrm{~T} 1$ \\
S9 & -3 & -5 & 6.0 & $2.33 \times 10^{-2}$ & $\mathrm{~T} 1$ \\
S10 & -3 & -5 & & $1.47 \times 10^{-2}$ & $\mathrm{~T} 2$ \\
\hline
\end{tabular}


Figures
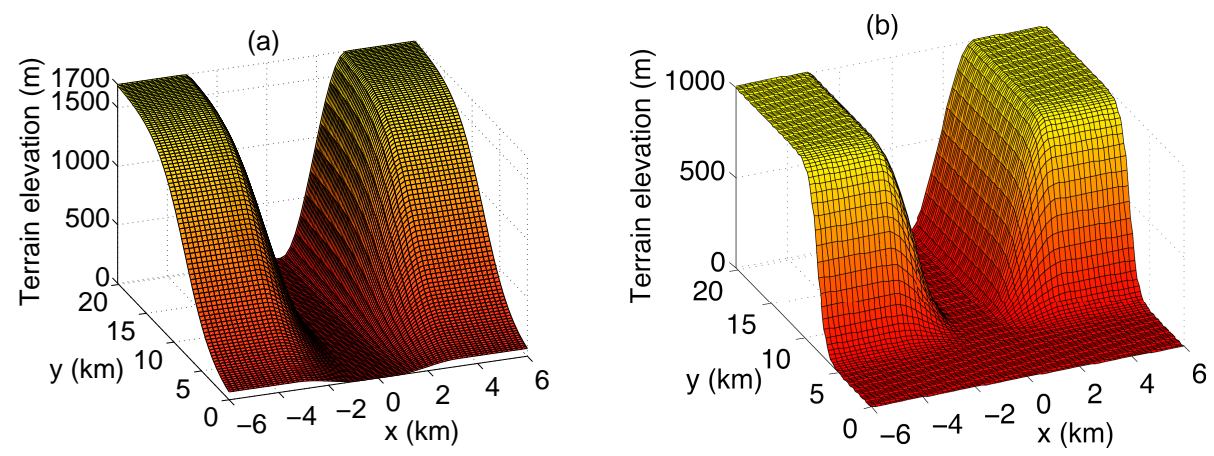

Fig. 1 Topography of the valleys (a) T1 and (b) T2 (see Section 2.2 for details). Note that the valley floor is flat 


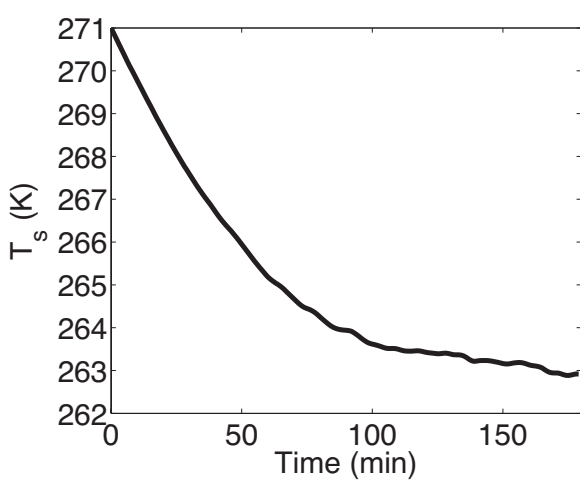

Fig. 2 Time series of the ground surface temperature $T_{S}$ half way down the slope at $y=15 \mathrm{~km}$ for simulation $\mathrm{S} 1$ 


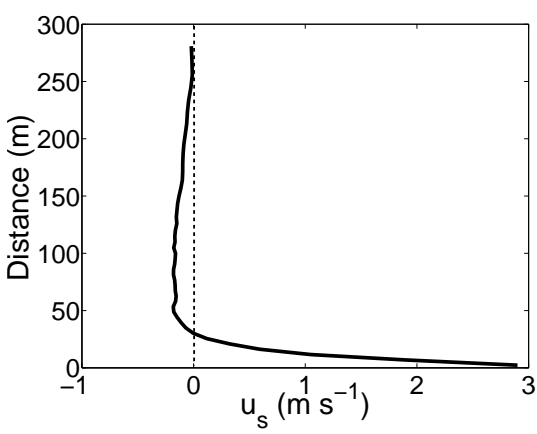

Fig. 3 Along-slope component $u_{s}$ of the katabatic wind versus distance along the vector normal to the sloping surface, for $y=7 \mathrm{~km}$ and $t=74 \mathrm{~min}$, at the $x$ location of maximum slope angle (i.e., half way down the slope), for simulation $\mathrm{S} 1$ 

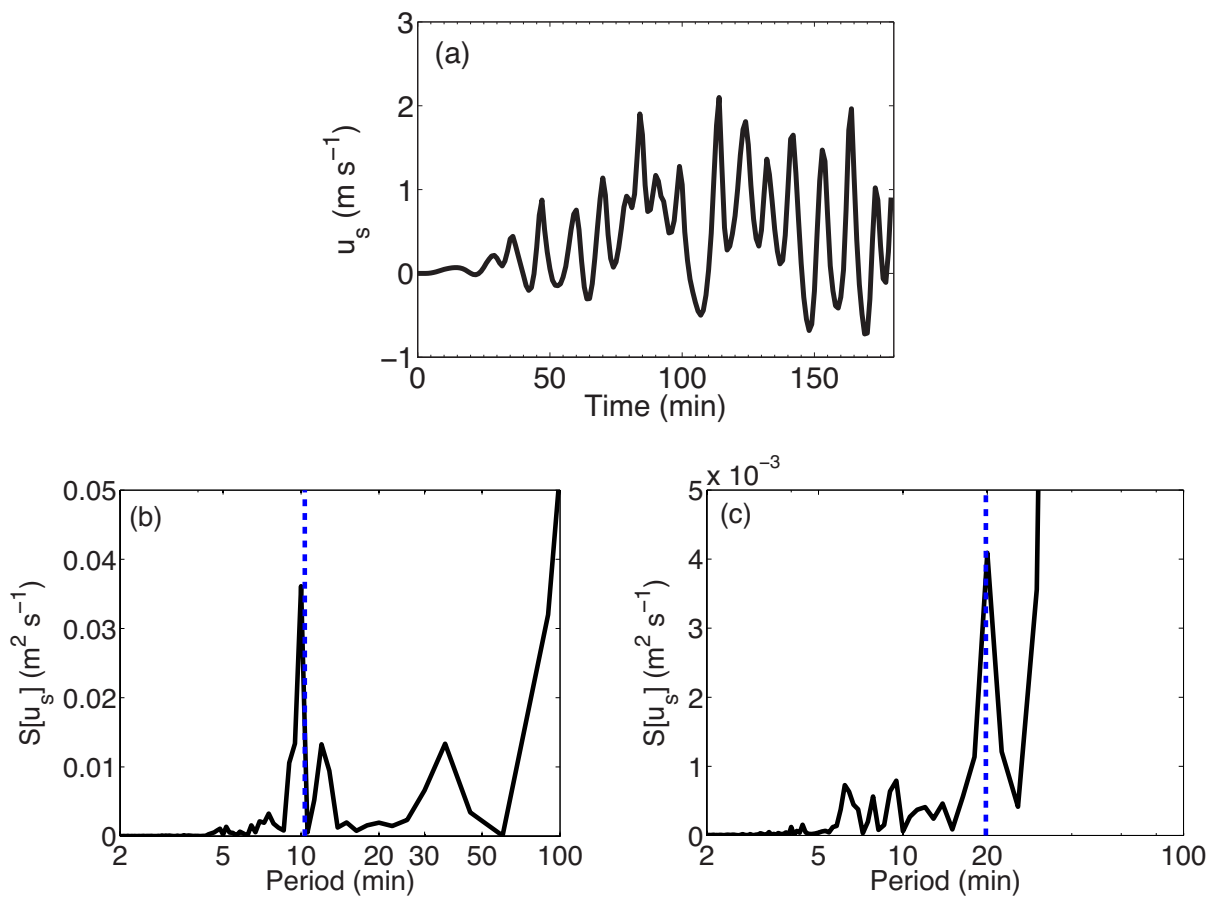

Fig. 4 For simulation S1: (a) Time series of the along-slope component of the wind $u_{s}$ at $x=-1.2 \mathrm{~km}$ and $y=15 \mathrm{~km}$ at $12.5 \mathrm{~m}$ above the ground surface; (b) Frequency velocity spectrum $S\left[u_{s}\right]$ of $u_{s}$ at the same location; (c) Frequency velocity spectrum $S\left[u_{s}\right]$ of $u_{s}$ at $x=-1.2 \mathrm{~km}$ and $y=7 \mathrm{~km}$ at $12.5 \mathrm{~m}$ above the ground surface. The blue dashed line in plots (b) and (c) indicates the period $2 \pi / \omega_{k}$ of the oscillations of the katabatic wind calculated using Equation 5 (see text for details) 


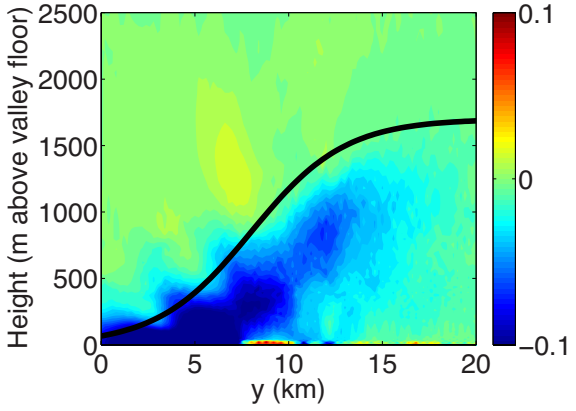

Fig. 5 For simulation S1: colour-filled contours of the along-valley component $v$ of the wind, in $\mathrm{m} \mathrm{s}^{-1}$, in a $(y, z)$ vertical cross section for $x=0 \mathrm{~km}$ and $t=45 \mathrm{~min}$. The black solid line indicates the height of the plateaus along the valley axis 

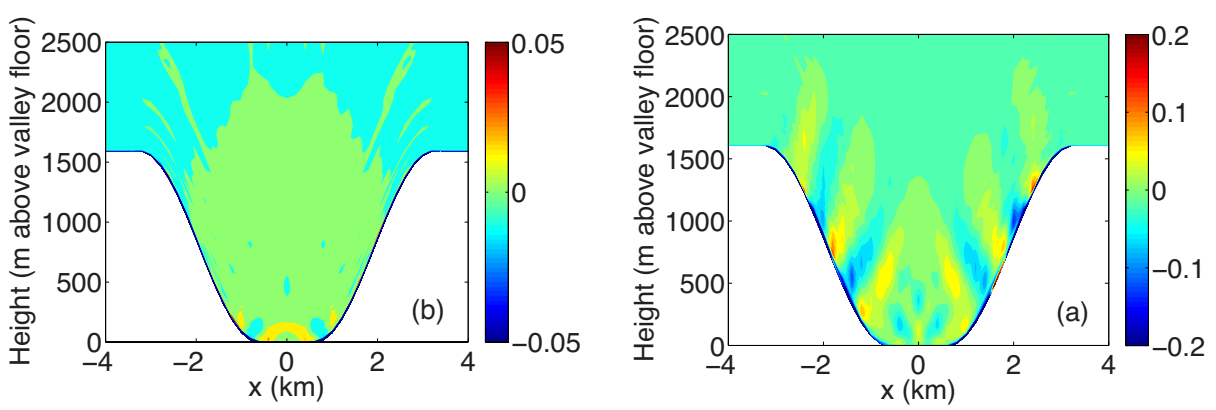

Fig. 6 For simulation S1: colour-filled contours of the vertical velocity component $w$, in $\mathrm{m} \mathrm{s}^{-1}$, in a vertical cross section for $y=15 \mathrm{~km}$ at (a) $t=25 \mathrm{~min}$ and (b) $t=45 \mathrm{~min}$ 


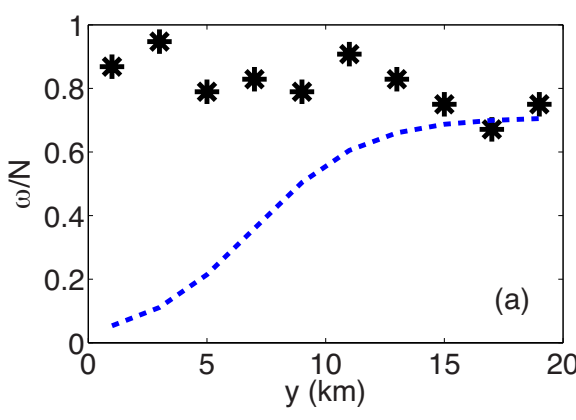

Fig. 7 For simulation S1: ratio of the IGW frequency $\omega_{w}$ to the buoyancy frequency $N(*)$, and of the frequency of the oscillations of the katabatic wind $\omega_{k}$ to

$N$ (blue dashed line) versus $y$ (i.e., along the valley axis) for $x=-0.6 \mathrm{~km}$ at an altitude of $2200 \mathrm{~m}$ 

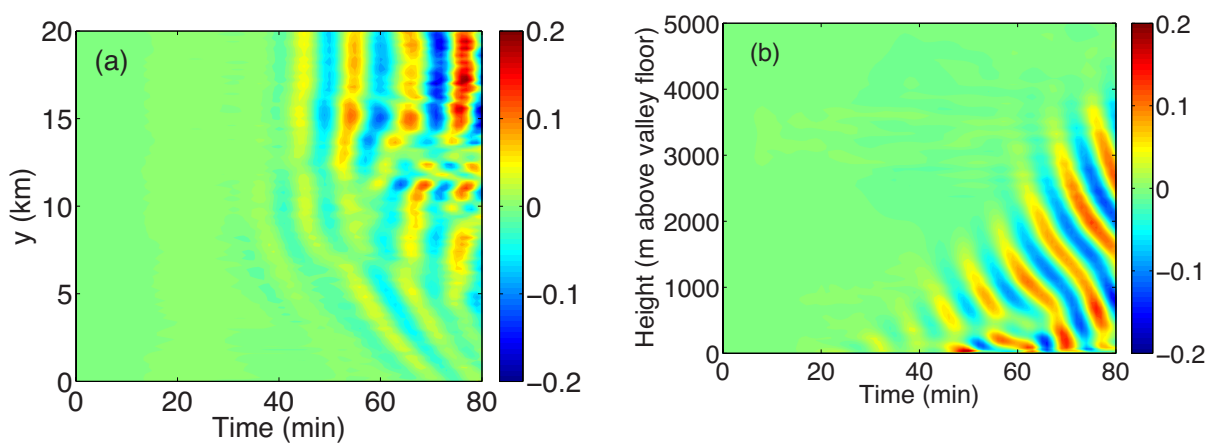

Fig. 8 For simulation S1: colour-filled contours of the vertical velocity component $w$, in $\mathrm{m} \mathrm{s}^{-1}$, (a) in a $(t, y)$ diagram for $x=-0.6 \mathrm{~km}$ at a height of $800 \mathrm{~m}$ above the valley floor and (b) in a $(t, z)$ diagram for $x=-0.6 \mathrm{~km}$ and $y=15 \mathrm{~km}$ 

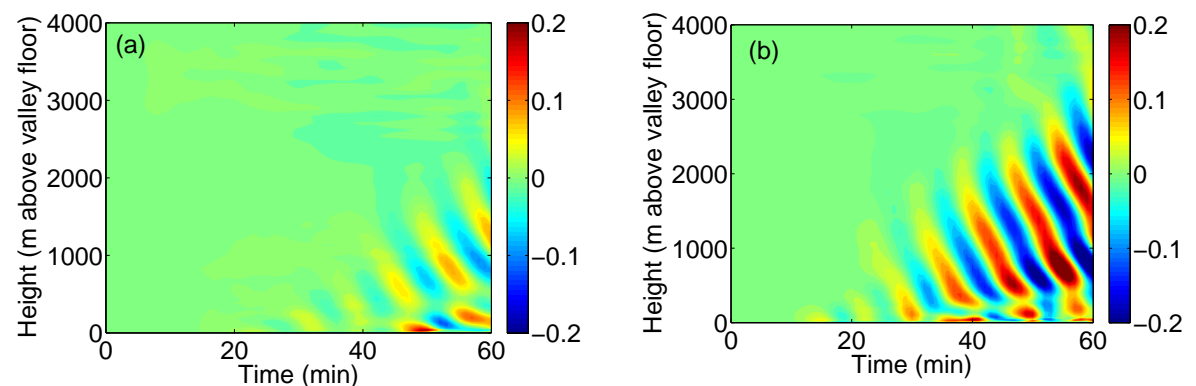

Fig. 9 Colour-filled contours of the vertical velocity component $w$, in $\mathrm{m} \mathrm{s}^{-1}$, in a $(t, z)$ diagram for $x=$ $-0.6 \mathrm{~km}$ and $y=15 \mathrm{~km}$ (a) for simulation S1 and (b) for simulation S5 


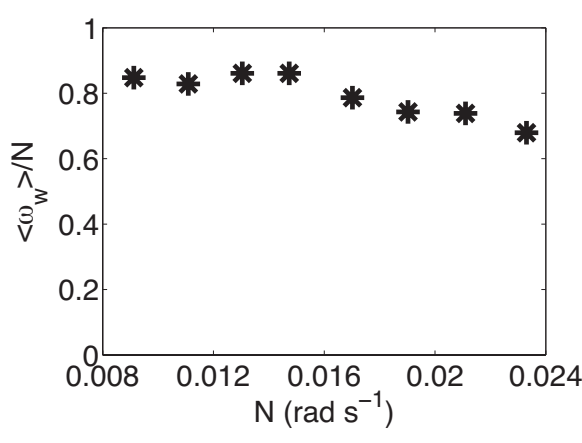

Fig. 10 Ratio of the mean value of the IGW frequency $\omega_{w}$ over $y$ (i.e., along the valley axis), denoted by $\left\langle\omega_{w}\right\rangle$, and the buoyancy frequency $N(*)$, for $x=-0.6 \mathrm{~km}$ at an altitude of $2200 \mathrm{~m}$, for each value of $N$ in the simulations $\mathrm{S} 2$ to $\mathrm{S} 9$ 

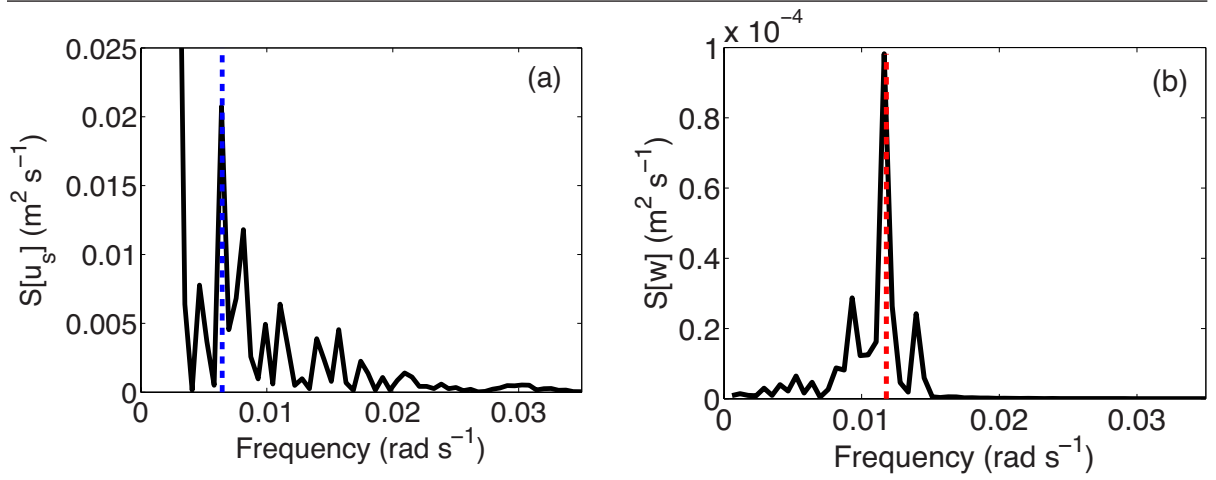

Fig. 11 For simulation S10: (a) Frequency velocity spectrum $S\left[u_{s}\right]$ of the along-slope component of the wind $u_{s}$ at $x=-1.2 \mathrm{~km}$ and $y=15 \mathrm{~km}$ at $12.5 \mathrm{~m}$ above the ground surface; (b) Frequency velocity spectrum $S[w]$ of the vertical component of the wind $w$ at $x=-1.2 \mathrm{~km}$ and $y=15 \mathrm{~km}$ at $4000 \mathrm{~m}$ above the ground surface. The blue dashed line in plot (a) indicates the frequency of the oscillations of the katabatic winds $\omega_{k}$ calculated using Equation 5. The red dashed line in plot (b) indicates the IGW frequency $\omega_{w} \approx 0.8 \mathrm{~N}$ 